\title{
A simple and effective pressure culture system modified from a transwell cell culture system
}

\author{
Xuerong Sun ${ }^{1,2}$, Xinguang Liu', Yuehong Zhang 2,3 , Xielan, Kuang ${ }^{2}, \mathrm{Bo} \mathrm{Lv}^{4 *}$ and Jian $\mathrm{Ge}^{2 *}$ \\ ' Institute of Aging Research, Key Laboratory for Medical Molecular Diagnostics of Guangdong Province, Guangdong Medical College, Dongguan 523808, China \\ 2 State Key Laboratory of Ophthalmology, Zhongshan Ophthalmic Center, Sun Yat-sen University, Guangzhou 510060, China. \\ ${ }^{3}$ Departments of Ophthalmology, First Municipal People's Hospital of Guangzhou, Affiliated Hospital of Guangzhou Medical College, Guangzhou, 510080, China. \\ ${ }^{4}$ Department of Emergency and Critical Care Medicine, Guangdong General Hospital, Guangdong Academy of Medical Sciences, Guangzhou 510080, China.
}

\begin{abstract}
Mechanical pressure plays an important role in many physiological and pathological processes. Mimicking the mechanical pressure present in vitro is necessary for related research, but usually requires expensive and complicated equipment. In this study we created a simple pressure culture system based on the transwell culture system. By cutting off the top rim of the transwell insert, the cells were compressed between the insert membrane and the well floor. The new pressure culture system was proven effective in that it induced cell morphological change, integrin $\beta 1$ upregulation, actin polymerization and growth change in rat retinal ganglion cells, human nasopharyngeal carcinoma cells and mice embryonic fibroblasts. Though the pressure value is immeasurable and inhomogeneous, the easily available culture system still provides a choice for the laboratories that do not have access to the better, but much more expensive pressure culture equipment.
\end{abstract}

Key words: Mechanical pressure; Cell culture; Transwell culture system; cytoskeleton.

\section{INTRODUCTION}

Mechanical pressure stimuli exists and plays important roles in many physiological and pathological processes [1-4]. In glaucoma, intracranial hypertension and solid tumors, the somatic cells are exposed to an elevated hydrostatic pressure $[5,6]$. In some physiological conditions, intervertebral discs and articular cartilage often bear a high mechanical burden, which probably includes both hydrostatic pressure and tensile strain. For vascular endothelial cells or the cells in the cavitas medullaris such as hemopoietic and mesenchymal stem cells, fluid shear stress has a great influence on proliferation, differentiation and other biological functions [7, 8]. Though hydrostatic pressure, mechanical strain and shear stress have different features, they all exert mechanical pressure on cells.

To facilitate the investigation of the biological role and mechanisms of mechanical pressure, it is necessary to establish this model in vitro. There has been a long history of pressure chamber inventions. Some pressure chambers were mounted into the microscopy to observe the depolymerization of spindle microtubules[9, 10], and some use the syringe - based pressure chamber to evaluate the ATP release from retina[11]. A tensegrity model has been established to explain the mechanism of cellular mechanotransduction, which considers cells and the extracellular matrix as a continuous network of fibers $[12,13]$.

Our lab has previously set up an elevated hydrostatic pressure model, which is mainly composed of an air-tight pressure chamber and a regulator which is connected to an additional power supply [14]. The model could provide a stable pressure in vitro, but it requires a huge incubator in which the pressure chamber is placed. Some other laboratories have established their own mechanical pressure models [15, 16], which are often complicated and not easily adoptable by others. Some commercial equipment also mimics mechanical pressure, but the equipment is often expensive and not available for many laboratories.

To establish an easily available pressure culture system, we made some modifications to the 6-well transwell culture system. The resulting hand-made pressure culture system is effective on rat retinal ganglion cell line 5 (RGC-5) cells, human nasopharyngeal carcinoma cell line (CNE-1) and mice embryonic fibroblasts (MEFs). This pressure culture system is simple and readily available for nearly every laboratory.

\section{MATERIALS AND METHODS}

\section{Cell culture}

The rat RGC-5 cell line was a gift from Prof. Zhikuan Yang in Zhongshan Ophthalmic Center, Sun Yat-sen University, and was cultured with high glucose Dulbecco's modified Eagle medium (DMEM medium) (Gibco, Grand Island, NY, USA) containing $10 \%$ fetal bovine serum (FBS; Hyclone, Logan, UT, USA). Mice MEFs were harvested from embryonic 13.5 days C57/BL6 mice and cultured with DMEM medium containing 10\% FBS. MEFs in 3 to 4 passages were used for this study. The CNE-1 cell line was cultured with 1640 medium (Gibco) containing 10\% FBS. All these cells were passaged by trypsin digestion, serum neutralization, centrifugalization and re-plating as usual. The usage of experiment animals and the protocol of the study were approved by the local ethics committee. 


\section{Design of the pressure culture system}

The pressure culture system was designed based on the transwell inserts (353102, BD Falcon) for 6-well plates. The bottom of the insert is sealed with cell-culture grade polyethylene terephthalate (PET) membranes (Figure 1). When placed into one well of the 6-well plate, the arcuated processes at the top of the insert lies right on the rim of the well. The insert and well together could be used as a non-contact coculture system [17] (Figure 1C).

To establish the pressure culture system, we cut off the arcuated processes at the top of the insert so that the whole insert descended and the membrane at the bottom of the insert pressed directly on the floor of the well (Figure 1B, 1D). When
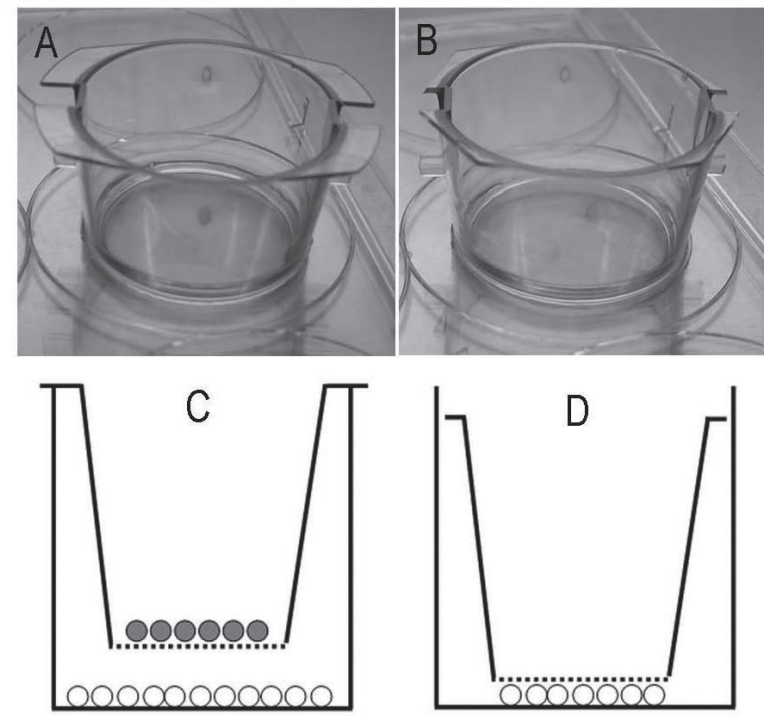

E

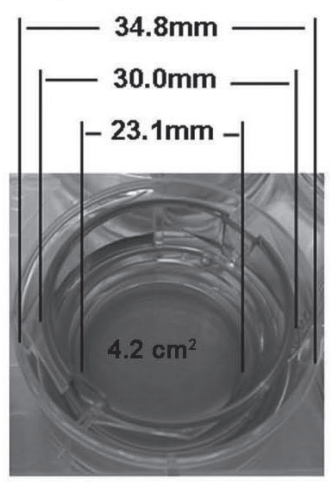

F

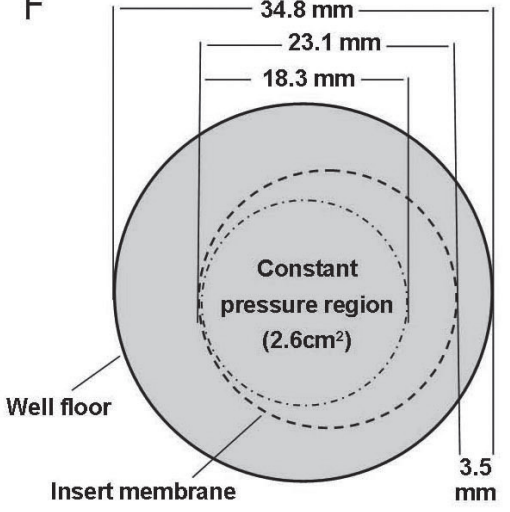

Figure 1: The pressure culture system modified from the transwell culture system. The transwell culture system is composed of an insert and a well, which can be used for co-culture of different types of cells $(\mathbf{A}, \mathbf{C})$. In figure $\mathbf{C}$ and $\mathbf{D}$, the dotted line at the bottom of the insert represents the semi-permeable PET membrane. To establish the pressure culture, the rim at the top of the insert was cut off (B) so that the insert was placed directly on the well floor, where the cells (blank circle) were mechanically compressed (D). The membrane area that could be used for the pressure culture was $4.2 \mathrm{~cm}^{2}$ (E). When the insert was randomly placed in the well, the $2.6 \mathrm{~cm}^{2}$ circle in the center of the well floor was consistently in the range of the pressure culture (F). cultured on the well floor, the cells are mechanically pressed by the PET membranes (Figure 1D).

In this study, to verify the pressure culture system $1 \times 10^{3}$ RGC-5, $1 \times 10^{3}$ CNE- 1 and $2 \times 10^{3}$ MEF suspensions were seeded into 6-well plates, within an approximately $19 \mathrm{~mm}$-diameter circle marked at the bottom center of the well with a pen. After allowing attachment for about 4-5 hours, the growth medium in each well was added up to $2.5 \mathrm{ml}$. When 24 hours had passed after cell seeding the modified inserts were put into the well, covering the circle line, to trigger the pressure culture. Meanwhile, $1.5 \mathrm{ml}$ growth medium was added into the insert. The growth medium in the insert was fully replaced with fresh medium every day, and a similar volume of growth medium was replaced in the normal control group. After pressure culture for 6, 12, 24 or 72 hours, RGC-5, CNE-1 and MEFs were harvested for real-time quantitative reverse transcription PCR (qRT-PCR) detection or cell counting.

\section{Staining of cell membrane with DiI}

DiI (1,1'-dioctadecyl-3,3,3', 3' -tetramethylindocarbocyanine perchlorate, C1036, Beyotime, China) was reconstituted with DMSO into $2 \mathrm{mM}$ stock solution. Before staining, DiI stock solution was diluted to $10 \mu \mathrm{M}$ with fresh growth medium. To stain the cells, the growth medium in the 6-well plates was removed and the fresh medium containing DiI was added. Then the plates were incubated in $37{ }^{\circ} \mathrm{C}$ for $20 \mathrm{~min}$. After washing with PBS solution 3 times, the cells were fixed with $4 \%$ paraformaldehyde for $15 \mathrm{~min}$ at room temperature. DAPI (C1002, Beyotime, China) was used to stain the nucleus. The cells were observed under a fluorescence microscope (Leica DM2500, Leica, Germany).

\section{Real-time quantitative reverse transcription PCR}

The procedure of qRT-PCR was similar to that we previously described with minimal modification [18]. Total RNA was extracted from RGC-5, CNE-1 and MEFs following the specification of RNeasy Mini Kit (Qiagen, Hilden, Germany). The likelihood of genomic DNA contamination was reduced with DNase I (Sigma, St. Louis, MO) treatment. cDNA was synthesized with a PrimeScript RT reagent kit (Takara, Tokyo, Japan). SYBR Premix Ex Taq (Takara) was used for quantitative PCR in an ABI Prism 7000 real-time PCR system. Glyceraldehyde-3- phosphate dehydrogenase (GAPDH) acted as the internal reference gene. The primer pairs for mice integrin $\beta 1$ were: forward TTGCCTTGCTGCTGATTTG, reverse TTTCACCCGTGTCCCACTT, product $105 \mathrm{bp}$; for rat integrin $\beta 1$ they were: forward AGTGAACAGCAACGGTGAAGC, reverse A GCA A G G C A G G C C A T A A G A, product $126 \mathrm{bp}$; and for human integrin $\beta 1$ they were: forward CAGTGAATGGGAACAACGAGG, reverse ATGCAAGGCCAATCAGAACAA, product $123 \mathrm{bp}$. The fold changes of integrin $\beta 1$ relative to $\mathrm{GAPDH}$ were calculated as previously described [18].

Immunofluorescence detection

To facilitate immunofluorescence detection, RGC-5, CNE-1 or MEFs were seeded onto a $22 \mathrm{~mm}$ round cover slip to initiate the pressure culture. After 1 or 3 days these cells were fixed with $4 \%$ paraformaldehyde for $15 \mathrm{~min}$ at room temperature. 
Expression of filamentous actin (F-actin) in these cells was detected following the specification of Actin-Tracker Green (Beyotime, China), which is FITC-labeled phalloidin. The tracker was 1:200 attenuated with PBS containing 0.1\% Triton X-100 and 4\% BSA, and then incubated with the cover slip for about $50 \mathrm{~min}$ at room temperature before detection by a fluorescence microscope (Leica DM2500).

\section{Cell proliferation detection}

Each well of a 6-well plate was seeded with $1 \times 10^{3}$ RGC-5 or CNE-1, or $2 \times 10^{3}$ MEFs cell suspension, up to 4 wells for each type, within a $19 \mathrm{~mm}$ circle as per the foregoing description. Of these, 2 wells were prepared for the pressure culture and 2 other wells served as the control. The pressure culture was initiated 24 hours after plating. After another 3 days the cells in each well were harvested with trypsin, and counted using a hemocytometer under an inverted microscope (Leica DMI3000 B, Leica). Since we noticed the attachment of some cells onto the transwell membrane in pressure culture, for the well of pressure culture the transwell membrane was also digested with trypsin and the liquid was harvested and counted together with the cell suspension of that well. The average cell number of the duplicate wells was regarded as the final result in that group. The cell number in each group was divided by the initial cell number to get the fold change. The experiment was repeated twice for each cell type.

\section{Statistical analysis}

Values were expressed as mean \pm standard error (number of observations). Analysis of variance (ANOVA) or Student's t-test was used to test for significant differences, and $P<0.05$ was taken to be significant.

\section{RESULTS}

\section{Parameter of the pressure culture system}

The pressure culture system was composed of a transwell insert and a 6-well plate with some modifications described in Methods (Figure 1A1D). The pressure came from the PET membrane attached at the bottom of the insert, which may exert mechanical tension on the cells.

The diameter of the PET membrane at the bottom of transwell inserts was $23.1 \mathrm{~mm}$ (Figure 1E- 1F). The cells were pressurecultured between PET membrane and the well floor; thus, the maximum area that could be achieved for pressure culture was only the area of the PET membrane, which is about $4.2 \mathrm{~cm}^{2}$ (Figure 1E). To facilitate seeding cells into the $4.2 \mathrm{~cm}^{2}$ circle, a line along the inner circle of the insert can be drawn at the bottom of the well.

The inner diameter of the well was 34.8 $\mathrm{mm}$, and the outer diameter of the insert top was $30.0 \mathrm{~mm}$ (Figure 1E). If the insert was placed randomly within the well, the mobilizable distance was $4.8 \mathrm{~mm}$. Therefore, a round area $(2.6$ $\mathrm{cm}^{2}$ ) with an $18.3 \mathrm{~mm}$ diameter in the center of the floor would be constantly within the range of pressure culture, wherever the inserts were placed (Figure $1 \mathrm{~F}$ ).

To get a preliminary impression of the pressure level, we detected the change of cell area after 3- or 7- day pressure culture. DiI is a kind of membrane-bound dye and was used to identify cell boundaries and thus to evaluate cell area. Normal culture did not induce obvious change on cell area. The results showed that after 3-day pressure culture, the cell area of CNE1 and MEFs decreased. The effect became more evident after 7 days. Typical pictures are shown in Figure 2. Similar results were observed in 2 independent experiments.

Morphology change and actin cytoskeleton rearrangement under pressure culture

Three kinds of cells, specifically rat RGC-5, human CNE-1 and mice MEFs, were selected to evaluate the pressure culture

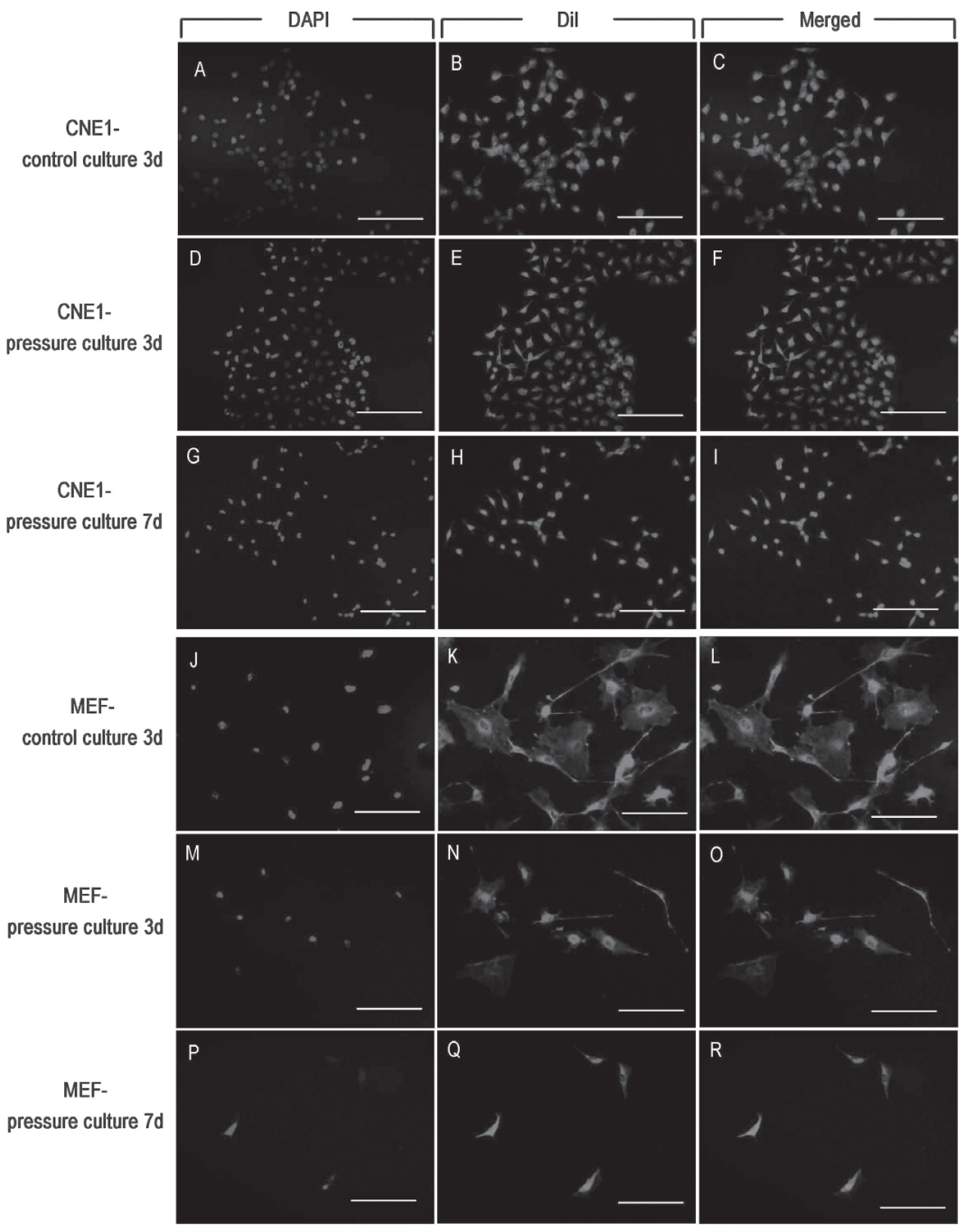

Figure 2: The effect of pressure culture on the cell area of CNE-1 and MEFs. Dil membrane dye was used to stain the cell membrane. It can be seen that after 3-day pressure culture, CNE-1 and MEFs became smaller than those in control culture. The effect became more evident after 7-day pressure culture. Bars represent $200 \mu \mathrm{m}$. 
system. Before pressure culture, RGC-5 cells had a short-rod shape with a few thin processes, CNE-1 cells were polygonal with short processes, and MEFs were long and spindle-shaped. After being pressure cultured for 1 or 3 days, the three types of cells showed similar changes, including a thinner body and more processes, often with a clearer cell boundary (Figure 3A).

Actin is an extensive-expression protein in somatic cells and also a sensor of mechanical stress [19, 20]. Phalloidin is a toxin from the toadstool which binds specifically to polymerized actin filaments rather than to actin monomers [21]. FITC-labeled phalloidin was used to detect the distribution of actin filaments. Before pressure culture, RGC-5, CNE-1 and MEFs cells all displayed evident green fluorescence by phalloidin staining, suggesting the universal existence of filamentous actin. After pressure culture for 1 or 3 days, expression of filamentous actin in all these cells became brighter and denser (Figure 3B), which might be from the result of the transition of globular actin into filamentous actin [22]. Similar phenomena were observed in three independent experiments.

The effect of pressure culture on integrin $\beta 1$ expression

Integrin $\beta 1$ plays an important role in sensing mechanical pressure or stress [23]. To verify the effect of the pressure culture system, expression of integrin $\beta 1$ was investigated by qRT-PCR before and after pressure culture. The results showed that after 6 hours of pressure culture, expression of integrin $\beta 1$ increased dramatically and peaked at 12 hours in RGC-5 (Figure 4A). After 12 hours, integrin $\beta 1$ expression gradually decreased. The expression of integrin $\beta 1$ in CNE-1 and MEFs cells also increased evidently upon pressure culture and peaked at 6 hours, after which the expression level declined. By 72 hours, the expression of integrin $\beta 1$ in MEFs was still significantly greater than that of the normal culture (Figure 4A).

To exclude the possible effect of PET membrane on integrin $\beta 1$ expression from the pressure culture system, $2.0 \times 10^{4}$ RGC-5, CNE-1 or MEFs cell suspensions were seeded onto the insert membrane placed on the well floor. Meanwhile, $4.6 \times 10^{4}$ RGC- 5 , CNE- 1 or MEFs cell suspensions were seeded into one well of a 6-well plate to achieve the same cell density. After 6 hours, qRTPCR detection showed that integrin $\beta 1$ expression was slightly less in the cells grown on the PET membrane than those grown on the well floor, but the difference was not significant (Figure 4B). The results suggested that upregulation of integrin $\beta 1$ in the pressure culture should be attributed to mechanical pressure, not the membrane substrate.

The effect of pressure culture on cell proliferation

Cells grown under mechanical pressure usually display a changed growth rate [3]. To assess the effect of the transwellbased pressure culture on cell proliferation, cells were counted after 3 days of pressure culture. Compared to control groups in the normal culture, 3-day pressure culture induced a significant increase in CNE-1 growth, along with an evident decrease in MEFs (Figure 5). Pressure culture also promoted the proliferation of RGC-5 slightly.

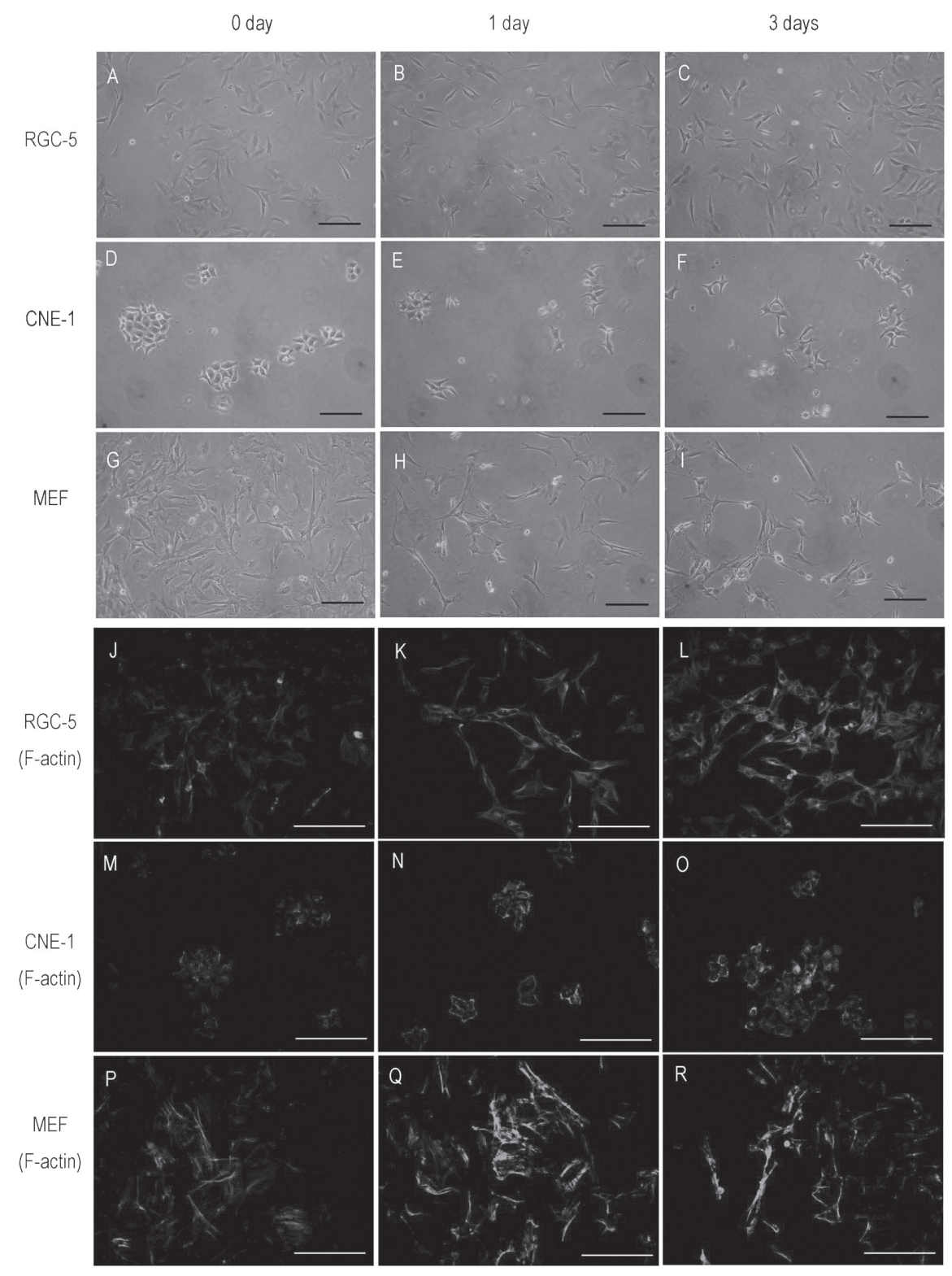

Figure 3: The morphology and actin cytoskeleton change in RGC-5, CNE-1 and MEFs under pressure culture. RGC-5, CNE- 1 and MEFs cells were pressure cultured for 3 days. Compared to the normal culture ( 0 day), 1 day or 3 days pressure culture induced obvious changes in these cells, including more processes, thinner and longer soma and clearer boundaries (A-I). For the actin cytoskeleton, pressure culture induced an obvious increase of filamentous actin in the three cell types (J-R). Bars represent $200 \mu \mathrm{m}$. 


\section{DISCUSSION}

This study showed that the pressure system was effective in that it induced morphological change, integrin $\beta 1$ upregulation, and actin polymerization in rat-, human-, and mouse-derived cells, which included both normal somatic cells and tumor cells. The morphological changes included a thinner, longer cell body and more processes. These changes were different than those produced under hydrostatic-pressure or shear-stress cultures [14, 24]. One reason accounting for this might be that the pressure exerted on the cell surface in this study was not as uniform as in hydrostatic-pressure culture, or not as unidirectional as in shear-stress cultures. Integrin $\beta 1$ upregulation was not long-lasting in this study, which might be due to a negative feedback regulation $[25,26]$.

The mechanical stimuli to which cells are usually subjected include hydrostatic pressure, shear stress and tensile strain [13]. In some reports, hydrostatic pressure induced actin
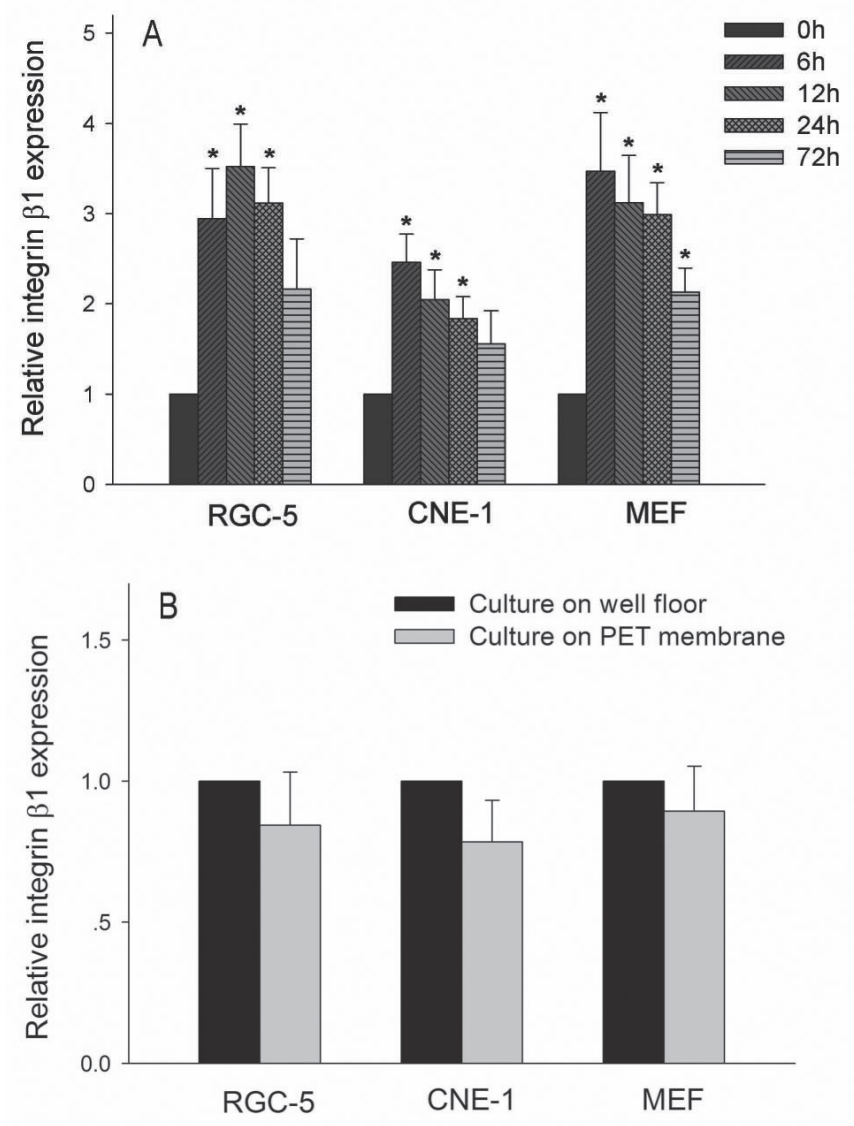

Figure 4: The effect of transwell-based pressure culture on integrin $\beta 1$ expression. The mRNA level of integrin $\beta 1$ was referred to GAPDH. A shows the relative integrin $\beta 1$ expression in RGC-5, CNE-1 and MEFs before and after pressure culture. Expression level before pressure culture $(0 \mathrm{~h})$ was defined as 1 . B shows the effect of membrane substrate on integrin $\beta 1$ expression. Cells were grown on the well floor or on the insert membrane for 6 hours before for integrin $\beta 1$ detection. The expression levels in well floor groups was defined as 1 . It should be noted that culture on membrane only decreased integrin $\beta 1$ expression slightly, but not significantly. Data were mean \pm standard error from 3 independent experiments. * represents $P<0.05$ vs. $0 \mathrm{~h}$. depolymerization [14, 27], while shear stress and tensile strain induced actin reorganization or polymerization [22, 28]. In this study, cells were quiescently compressed between two objects, which induced an obvious increase of filamentous actin. Therefore, tensile strain might be the main stimulus in our pressure culture system.

The pressure culture system was modified from the transwell system of Becton, Dickinson and Company. The cells were mechanically compressed between the insert membranes and the well floor. Based on the principle, the transwell systems from other companies such as Coring, Millipore or Costar could also be easily modified into pressure culture systems by cutting off the rim of the insert so that the insert is directly placed on the well floor.

In this study, the membrane of the insert was made of polyethylene terephthalate (PET), which is transparent to facilitate observation. There are also insert membranes made of polycarbonate. These membrane materials are all cellculture grade and appropriate for pressure cultures. Different membrane pore sizes can be chosen, such as $0.4 \mu \mathrm{m}, 1 \mu \mathrm{m}$ or $3 \mu \mathrm{m}$. To guarantee the pressure effect, the membrane pore size should be smaller than the cell diameter, which depends on cell type, cell cycle progression and cell viability, among other factors.

The advantages of this pressure culture system are obvious. First, it is easy and available for every laboratory if they meet the conditions for cell culture. Second, the insert membrane is permeable to gasses such as carbon dioxide and oxygen, as well as molecular substances such as proteins; thus the condition of the pressure culture is almost completely the same as that of a normal culture, except for the pressure.

The shortcomings of the pressure culture system are also equally obvious. Most significantly, the pressure exerted on cells is inhomogeneous, immeasurable, and dynamically changing due to the growth of cells. Although we observed a decrease in cell area of CNE-1 and MEFs under pressure

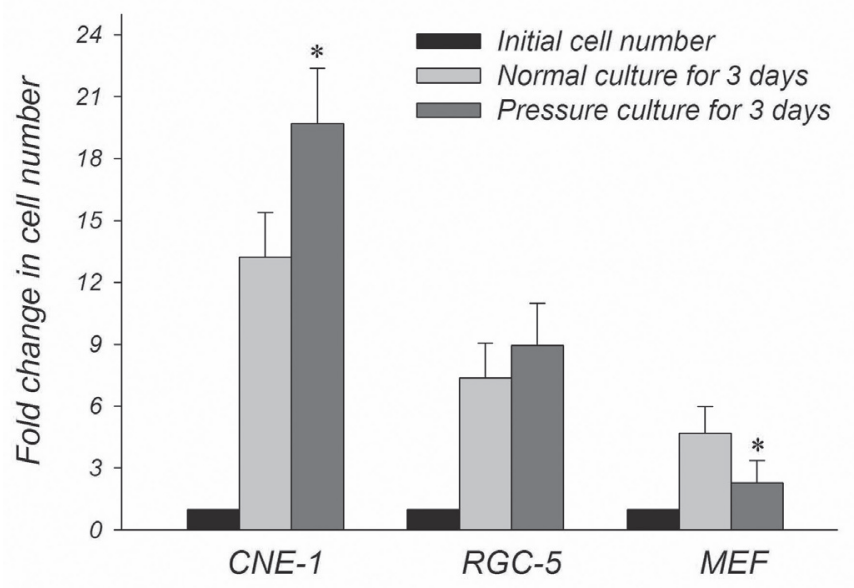

Figure 5: The effect of pressure culture on the proliferation of RGC-5, CNE- 1 and MEFs. The initial cell number of each cell type $\left(1 \times 10^{3}\right.$ RGC-5, $1 \times 10^{3}$ CNE- 1 and $2 \times 10^{3}$ MEFs) was defined as 1. Normal culture served as the control. 3-day pressure culture promoted the growth of CNE-1, while inhibited MEFs significantly. Data were obtained from 3 independent experiments. * represents $P<0.05$ vs. normal culture. 
culture, the exact pressure level was unknown. Furthermore, the pressure exerted on each cell might be different. Many factors could affect the pressure level. The factors arising from cells include cell size, cell density and cell distribution, among others. The membrane material, membrane pore size, and nominal pore density car also influence the pressure level. Even the position in which the individual cell seeded or the placement of the insert might change the pressure level. Additionally, the pressure culture system can be used only for adherent cells, not for suspension cells.

Mechanical stimuli have great effects on cell proliferation. Elevated interstitial fluid pressure or mechanical strain may either increase or decrease cell growth, depending on cell type, stimuli amplitude, stimuli frequency, and other factors [3, 29]. The pressure culture in our study induced growth promotion on CNE-1 and RGC-5, while inducing evident inhibition on MEFs. It was reported that another kind of mouse fibroblast, 3T3-L1 grew faster under pressure of $5 \mathrm{MPa}$ than at normal atmospheric pressure[30]. The difference might be due to the reason that the influence of mechanical pressure on cell proliferation is cell-type dependent[3]. 3T3-L1 is an established cell line and grows faster than MEFs. In current study, the MEFs were seeded at low density, which might also enhance the sensitivity to pressure stimulus.

In summary, we established an easily available pressure culture system which is effective for various kinds of cell types and can exert obvious influence on cell morphology and biological functions. Mechanical strain probably is the main component of the pressure.

\section{ACKNOWLEDGMENTS}

This work was supported by the National Natural Science Foundation of China $(81170846,81170327)$, the Fundamental Research Funds of State Key Laboratory of Ophthalmology (QN-1), the Medical Scientific Research Foundation of Guangdong Province (A2012420) and the Science \& Technology Innovation Fund of Guangdong Medical College (STIF201102).

\section{REFERENCES}

1. AGHA R, et al. (2011) A review of the role of mechanical forces in cutaneous wound healing. J Surg Res. 171(2): p. 700-8.

2. OH S, et al. (2010) Role of elevated pressure in TRAIL-induced apoptosis in human lung carcinoma cells. Apoptosis. 15(12): p. 1517-28.

3. DIRESTA GR, et al. (2005) Cell proliferation of cultured human cancer cells are affected by the elevated tumor pressures that exist in vivo. Ann Biomed Eng. 33(9): p. 1270-80.

4. LEVENTAL KR, et al. (2009) Matrix crosslinking forces tumor progression by enhancing integrin signaling. Cell. 139(5): p. 891-906.

5. LUNT SJ, et al. (2008) I nterstitial fluid pressure, vascularity and metastasis in ectopic, orthotopic and spontaneous tumours. BMC Cancer. 8: p. 2.

6. CAPRIOLI J and VARMA R (2011) I ntraocular pressure: modulation as treatment for glaucoma. Am J Ophthalmol. 152(3): p. 340-344 e2.
7. THOMPSON WR, RUBIN CT and RUBIN J (2012) Mechanical regulation of signaling pathways in bone. Gene.

8. THILO F, et al. (2012) Pulsatile atheroprone shear stress affects the expression of transient receptor potential channels in human endothelial cells. Hypertension. 59(6): p. 1232-40.

9. SALMON ED and ELLIS GW (1975) A new miniature hydrostatic pressure chamber for microscopy. Strain-free optical glass windows facilitate phasecontrast and polarized-light microscopy of living cells. Optional fixture permits simultaneous control of pressure and temperature. J Cell Biol. 65(3): p. 587602 .

10. SALMON ED (1975) Pressure-induced depolymerization of spindle microtubules. I. Changes in birefringence and spindle length. J Cell Biol. 65(3): p. 603-14.

11. REIGADA D, et al. (2008) Elevated pressure triggers a physiological release of ATP from the retina: Possible role for pannexin hemichannels. Neuroscience. 157(2): p. 396-404.

12. INGBER DE (2006) Cellular mechanotransduction: putting all the pieces together again. Faseb J. 20(7): p. 811-27.

13. MYERS KA, et al. (2007) Hydrostatic pressure sensation in cells: integration into the tensegrity model. Biochem Cell Biol. 85(5): p. 543-51.

14. YANG X, et al. (2011) Elevated pressure downregulates ZO-1 expression and disrupts cytoskeleton and focal adhesion in human trabecular meshwork cells. Mol Vis. 17: p. 2978-85.

15. JU WK, et al. (2009) Elevated hydrostatic pressure triggers release of OPA1 and cytochrome $C$, and induces apoptotic cell death in differentiated RGC-5 cells. Mol Vis. 15: p. 120-34.

16. LEI Y, et al. (2011) In vitro models for glaucoma research: effects of hydrostatic pressure. Invest Ophthalmol Vis Sci. 52(9): p. 6329-39.

17. SUN X, et al. (2011) E13.5 retinal progenitors induce mouse bone marrow mesenchymal stromal cells to differentiate into retinal progenitor-like cells. Cytotherapy. 13(3): p. 294-303.

18. SUN X, et al. (2009) Gene expression and differentiation characteristics in mice E13.5 and E17.5 neural retinal progenitors. Mol Vis. 15: p. 2503-14.

19. GARDEL ML, et al. (2010) Mechanical integration of actin and adhesion dynamics in cell migration. Annu Rev Cell Dev Biol. 26: p. 315-33.

20. STRICKER J, FALZONE T and GARDEL ML (2010) Mechanics of the F-actin cytoskeleton. J Biomech 43(1): p. 9-14.

21. COOPER JA (1987) Effects of cytochalasin and phalloidin on actin. J Cell Biol. 105(4): p. 1473-8.

22. CIPOLlA MJ, GOKINA NI and OSOL G (2002) Pressure-induced actin polymerization in vascular smooth muscle as a mechanism underlying myogenic behavior. Faseb J. 16(1): p. 72-6.

23. MCILHENNY SE, et al. (2010) Linear shear conditioning improves vascular graft retention of adipose-derived stem cells by upregulation of the alpha5beta1 integrin. Tissue Eng Part A. 16(1): p. 245-55.

24. THOUMINE O, NEREM RM and GIRARD PR (1995) Oscillatory shear stress and hydrostatic pressure modulate cell-matrix attachment proteins in cultured endothelial cells. In Vitro Cell Dev Biol Anim. 31(1): p. 45-54.

25. HUVENEERS S and DANEN EH (2009) Adhesion signaling - crosstalk between integrins, Src and Rho. J Cell Sci. 122(Pt 8): p. 1059-69.

26. SHYY JY and CHIEN S (1997) Role of integrins in cellular responses to mechanical stress and adhesion. Curr Opin Cell Biol. 9(5): p. 707-13.

27. TOKUDA S, et al. (2009) Hydrostatic pressure regulates tight junctions, actin cytoskeleton and transcellular ion transport. Biochem Biophys Res Commun. 390(4): p. 1315-21.

28. ARNSDORF EJ, et al. (2009) Mechanically induced osteogenic differentiation-the role of RhoA, ROCKII and cytoskeletal dynamics. J Cell Sci. 122(Pt 4): p. 546-53.

29. GAYER CP and BASSON MD (2009) The effects of mechanical forces on intestinal physiology and pathology. Cell Signal. 21(8): p. 1237-44.

30. KOYAMA S, et al. (2005) Effects of the piezo-tolerance of cultured deepsea eel cells on survival rates, cell proliferation, and cytoskeletal structures. Extremophiles. 9(6): p. 449-60. 